\title{
Occupant monitoring for facility management using Radio Frequency Identification
}

\section{Farhan Manzoor Ahmed Khan}

School of Science, Engineering and Food Science - Department of Civil and Environmental Engineering, UCC

\section{Introduction}

Mankind's rapidly increasing advancements in different industrial sectors demand a great price of environmental impact and climate change in return, specifically in the buildings and construction industry. The largest source of greenhouse gas emissions and energy consumption worldwide are buildings, estimated to account for almost $48 \%$ of all such emissions. Energy-related Carbon Dioxide $\left(\mathrm{CO}_{2}\right)$ counts for about $82 \%$ of all greenhouse gases emitted by human activities. This total energy consumption translates to approximately 3.5 Billion Euros per annum. According to a report from the United Nations Environment Programme, the right mix of appropriate government regulations, greater use of energy-saving technologies and user behavioural changes can substantially reduce $\mathrm{CO}_{2}$ emissions from buildings. The Energy Performance of Buildings Directive places an onus on all EU member states to rate the energy performance of buildings in a Building Energy Rating certificate which is effectively an energy label required at the point of rental or sale of a building.

These figures and percentages strongly imply alternatives to proper energy management in buildings for minimising $\mathrm{CO}_{2}$ emissions. Efficient energy and environmental performance management systems for residential buildings do not exist; such systems consist of an ad-hoc integration of wired building management and monitoring for non-residential buildings. Goal of the Information and Communication Technologies (ICT) for Sustainable and Optimised Building Operation project, along with its strategic partners is to improve asset management while supplementing efforts to meet the requirements of the Kyoto protocol.

The project envisages to:

enhance the management of large-scale, complex networks, services, and mobile users by introducing new network and management technologies;

develop frameworks and algorithms to support collaborative support and contributions for energy efficient buildings; 
support different end-to-end networks and services through sensors and Radio Frequency Identification (RFID) hardware.

\section{Research in Occupant Monitoring}

Previous research has concluded that an excessive energy usage is accounted for due to behaviour of occupants and their energy usage. There is a strong need to monitor this behaviour and analyse building energy consumption demands and requirements on its basis. Until now, no standard method exists to determine occupant behaviour within buildings; different approaches are used based on a building's size, type, and functional use. Some of these approaches include fixed and preset supply of energy, whereas others perform requirements analysis based on occupant requests. We propose a methodology of continuously monitoring occupant density patterns to estimate energy usage requirements for optimal thermal comfort and cost savings. By definition, 'Occupant Density gives the number of people present in a building space at a particular time', whereas, an 'Occupant Pattern represents the use of a building space over a specific period of time'; this can also be considered as the collection of occupant density data over a specific time period. Essentially, based on these two parameters, 'optimal' thermal comfort is envisaged to be achieved through controlling the Heating, Ventilation, and Air-Conditioning (HVAC) module of the Environmental Research Institute at University College Cork. Operation and maintenance of building components along with thermal comfort needs is one of the most important and challenging goals of a building's Facilities Management (FM) team; the only question by facilities managers is "How to?". Occupant patterns are valuable in helping make predictive building control decisions because they can provide peak and off-peak energy usage timings.

\section{Radio Frequency Identification (RFID)}

Different technologies are available that can be combined with building operation and FM activities, one in particular is Radio Frequency Identification (RFID). As part of the Networked Embedded System project, we implement fixed and mobile location-based sensing solutions of occupants and inventory items management in FM application scenarios using RFID. Occupant tracking and localisation is achieved through a passive RFID gateway portal setup. The portal houses four RFID antennas that read the unique IDs of tags passing through and transfer it to the RFID reader. Passive RFID tags, having no battery, are form factored as cards/badges and are handed out to occupants or users of a specific building facility and are to be carried at all times. Ultra High Frequency (UHF) RFID technology is generally not used for personnel tracking and localisation due to problems of UHF not being able to penetrate through liquids and metals. Despite this, long-range and high data 
rates of UHF offer an interest and challenge to use these low cost RFID tags in our application scenarios. In order to ensure effective and reliable tag readings, specific rules or constraints are to be followed, therefore, users are trained in appropriate tag placements and their usage.

\section{Workflow Strategy}

Raw Data collected from RFID is stored into a local database where it is filtered and applied with business logic in order for unique IDs to correspond to specific personnel or items, thereby being translated into meaningful data. The central database is accessible via the Building Management System (BMS) which in turn controls the HVAC and other building components. The facilities manager has access to the BMS in order to take control decisions based on the data gathered from Wireless Sensor Networks and RFID. This can also be an automated process through making the system learn and predict from previous occupant pattern trends. Thermal comfort in this way is based on the dynamic flow of entrance and exits of users within a building facility.

One might argue that this can be achieved through other tracking and tracing technologies, e.g. Passive Infrared sensors. Another argument may concern the privacy and security of individuals. The application diversity of using RFID is not just to count the number of occupants but to also identify them for emergency evacuation scenarios. In such scenarios, prioritised evacuation can be executed for the elderly, young, or disabled. In the case of fire emergencies, fire fighters can be updated of the exact number and identity of people trapped inside a building under fire.

\section{Conclusion}

An essential source of $\mathrm{CO}_{2}$ emissions within buildings is due to unnecessary heating and ineffective or absent methods of heat recovery. Using RFID technology, occupant monitoring within buildings is performed in order to determine their density and patterns. This accounts for analysis of their usage behaviour towards energy resources in order to provide optimal thermal comfort through predictive building control decisions. It is important to properly utilize such resources based on needs and requirements in order to have effective energy management throughout the building. Eventually, people operate and consume buildings' energy resources and it is only our choice to be responsible towards the amount of $\mathrm{CO}_{2}$ emission and hence, towards climate change and environmental impact.

Thanks to my supervisor, Professor Karsten Menzel, colleagues, and members of the Networked Embedded Systems (NEMBES) and the Informatics Research Unit for Sustainable Engineering (IRUSE) research groups at the Department of Civil and Environmental Engineering, University College Cork. 